\title{
Robust guaranteed performance PID controller design for non-minimum phase plants
}

\author{
Štefan Bucz ${ }^{*}$, Alena Kozáková**, Vojtech Veselý*
}

\begin{abstract}
The paper presents a new original robust PID design method for non-minimum phase plants to achieve closed-loop performance prescribed by the process technologist in terms of settling time and maximum overshoot, respectively. The proposed design procedure has two steps: first, the uncertain system is identified using external harmonic excitation signal with frequency, second, the controller of the nominal system is designed for specified gain margin. A couple of parameters is obtained from the time domain performance specification using quadratic regression curves, the so-called performance Bparabolas so, as to simultaneously satisfy robust closed-loop stability conditions. The main benefits of the proposed method are universal applicability for systems with both fast and slow dominant dynamics as well as performance specification using time domain criteria. The proposed PID design method has been verified on a set of benchmark systems.
\end{abstract}

K e y w o r d s: robust PID controller design, guaranteed performance, robust stability, robust performance, unstable zero, excitation level

\section{Introduction}

Difficulties in control of the class of non-minimum phase plants $F(s)=(1-\beta s) /(1+T s)^{n}$ with an unstable (positive) zero $z=1 / \beta$ even for small values of $\beta$ are well known as well as growing control complexity with increasing $\beta$, [1]. This problem is encountered in the control of continuous stirred tank reactors, flexible robotic arms, servomechanisms, level control systems of steam boilers as well as in processes of increasing power of water turbines. A similar problem occurs when a time delay is approximated using a higher order Padé approximation. Unstable zeros decrease closed-loop bandwidth, deteriorate its dynamics and robustness against disturbances [2].

Control theory provides various controller design approaches for systems with an unstable zero, eg root locus method, LQG theory, IMC approach, and fuzzy control. A comprehensive survey on control of systems with unstable zero based on the above approaches can be found in $[3-21]$.

There are several tuning methods for systems with unstable zero described by typical models. For FOPTD models (First Order Plus Time Delay) with an unstable zero the method by Sree and Chidambaram [22] can be applied. Using the first method, an ideal PI controller is designed, the second method yields a real PI controller in series with a first-order filter; in both cases the performance is not specified. For SOPTD models (Second Order Plus Time Delay) with an unstable zero an ideal PID controller guaranteeing a maximum peak of the sensitivity $M_{s}=1.5$ can be designed using the method by Wang et al [23]. The approach proposed by Huang et al, [24] yields coefficients of a non-interactive PID controller based on the two-degree of freedom structure [25] with a filtered derivative part and non-specified performance. Tuning the serial PI-PD controller according to Poulin and Pomerleau [26] guarantees a phase margin $\varphi_{M} \geq 65$ (and according to O'Dwyer [27] both the gain margin $A_{m}=2$ and phase margin $\varphi_{M}=45^{\circ}$. In the $\lambda$-tuning method according to Chien [28] the expected settling time is limited by the time delay, however with no possibility to closed specify the performance. Therefore, there is a natural demand for such controller design methods that are universally applicable for a broad range of systems and guarantee specified performance even for systems with varying parameters. The presented paper follows this idea.

The new proposed method is applicable for control of linear single-input-single-output non-minimum phase systems even with unknown mathematical model with unstructured uncertainties. The control objective is to provide required nominal closed-loop settling time $t_{\text {reg }}$ and maximum overshoot $\eta_{\max }$, respectively.

The key idea of guaranteeing specified $t_{\text {reg }}$ and $\eta_{\max }$ was to generalize the relations $t_{\mathrm{reg}}=f\left(\omega_{e}\right)$ and $\eta_{\max }=$ $f\left(G_{M}\right)$ known from the Reinisch formulas [29] where their validity is restricted just for 2 -nd order systems. Their generalization for systems of arbitrary order has proved a quadratic dependence on two parameters in both cases: $t_{\text {reg }}=f\left(\omega_{e}, G_{M}\right)$ and $\eta_{\max }=f\left(\omega_{e}, G_{M}\right)$; their graphical representation the so-called $B$-parabolas is an important design tool that enables choosing such a pair of parameters $\left(G_{M}, \omega_{e}\right)$ that guarantee closed-loop robust perfor-

* Institute of Robotics and Cybernetics, Slovak University of Technology in Bratislava, Slovakia, stefan.bucz@stuba.sk, vojtech.vesely@stuba.sk, ** Institute of Automotive Mechatronics, Slovak University of Technology in Bratislava, Slovakia, alena.kozakova@stuba.sk 
mance specified in terms of $t_{\text {reg }}$ and $\eta_{\max }$. In this way the closed-loop step response of the plant with an unstable zero and unstructured uncertainty can be systematically shaped.

\section{Uncertain plant identification using a harmonic excitation input}

Consider the multipurpose control loop in Fig. 1, where the control $u(t)$ is generated using a three-position switch $S W$. A PID controller $F_{R}(s)$ is to be designed such that the controlled output $y(t)$ properly tracks the reference $w(t)$. Transfer function of the uncertain nonminimum phase system is $F(s)$.

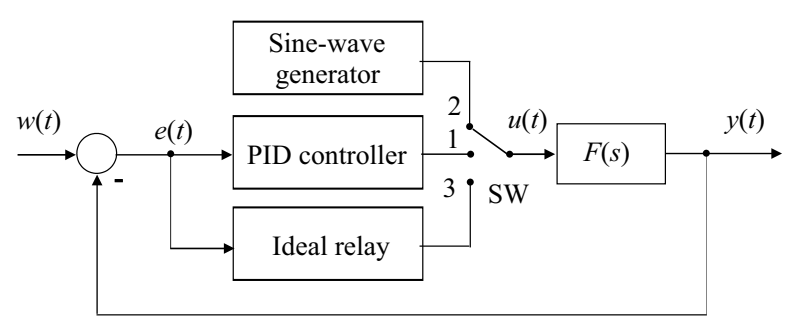

Fig. 1. Multipurpose loop for the proposed harmonic excitationbased robust PID design method

If we apply a zero setpoint $w(t)=0$ and switch $S W$ into the position 2 , the sinusoid signal generator excites the unknown uncertain controlled system $F(s)$ using the signal $u(t)=U_{e} \sin \left(\omega_{e} t\right)$ The corresponding response is a harmonic signal $y(t)=Y_{e} \sin \left(\omega_{e} t+\varphi\right)$ with a phase shift $\varphi(\omega)$. From the ratio of amplitudes $Y_{e}\left(\omega_{e}\right) / U_{e}\left(\omega_{e}\right)$ obtained from measurements of $u(t)$ and $y(t)$ we can calculate location of the related point $\mathcal{F}\left(j \omega_{e}\right)$ on the Nyquist plot of the unknown controlled system

$$
\mathcal{F}\left(j \omega_{e}\right)=F\left(\omega_{e}\right) e^{j \varphi\left(\omega_{e}\right)}=\frac{Y\left(\omega_{e}\right)}{U\left(\omega_{e}\right)} e^{j \varphi\left(\omega_{e}\right)} .
$$

By performing multiple identification experiments for individual changes in uncertain parameters of the unknown system under the same frequency $\omega_{e}$ of the excitation signal we obtain a set of $N=2^{p}$ identified points $\mathcal{F}_{i}, i=1 \ldots N$ corresponding to $N$ Nyquist plots of the unknown uncertain system

$$
\mathcal{F}_{i}\left(\omega_{e}\right)=\left(\begin{array}{c}
F_{1}\left(\omega_{e}\right) \\
F_{2}\left(\omega_{e}\right) \\
\ldots \\
F_{N}\left(j \omega_{e}\right)
\end{array}\right) \exp \left(\begin{array}{c}
j \varphi_{1}\left(\omega_{e}\right) \\
j \varphi_{2}\left(\omega_{e}\right) \\
\ldots \\
j \varphi_{N}\left(\omega_{e}\right)
\end{array}\right)
$$

above $p$ denotes the number of varying process quantities $F(s)$. The nominal point position

$$
\mathcal{F}_{0}\left(j \omega_{e}\right)=F_{0}\left(\omega_{e}\right) e^{j \varphi_{0}\left(\omega_{e}\right)}
$$

can be calculated according to [30]

$$
\begin{aligned}
F_{0}\left(\omega_{e}\right) & =\frac{1}{N}\left[\left(\sum_{i=1}^{N} F_{i}\left(\omega_{e}\right) \cos \varphi_{i}\right)^{2}\right. \\
+ & \left.\left(\sum_{i=1}^{N} F_{i}\left(\omega_{e}\right) \sin \varphi_{i}\right)^{2}\right]^{1 / 2}, \\
\varphi_{0}\left(\omega_{e}\right) & =\operatorname{arctg} \frac{\sum_{i=1}^{N} F_{i}\left(\omega_{e}\right) \sin \varphi_{i}}{\sum_{i=1}^{N} F_{i}\left(\omega_{e}\right) \cos \varphi_{i}} .
\end{aligned}
$$

Position of the worst-case point

$$
\mathcal{F}_{N}\left(j \omega_{e}\right)=F_{N}\left(\omega_{e}\right) e^{j \varphi_{N}\left(\omega_{e}\right)}
$$

can be determined using the following expressions [30] From here the variable $\omega_{e}$ will be omitted for simplicity if not on clarity account

$$
\begin{gathered}
F_{N}=\sqrt{F_{0}^{2}+R_{G}^{2}-2 F_{0} R_{G} \cos \Delta \varphi} \\
\varphi_{N}=\varphi_{+} \Delta \varphi_{0 N}
\end{gathered}
$$

where $\Delta \varphi_{0 N}$ is a phase difference between the worst-case point $\mathcal{F}_{N}$ and the nominal point $\mathcal{F}_{0}$ of the uncertain system

$$
\begin{gathered}
\Delta \varphi_{0 N}=\arccos \frac{F_{0}-R_{G} \cos \Delta \varphi}{F_{N}}, \\
\Delta \varphi=\arccos \frac{F_{0}+\cos \varphi_{0}}{\sqrt{F_{0}^{2}+2 F_{0} \cos \varphi_{0}+1}} .
\end{gathered}
$$

Position of the nominal point $\mathcal{F}_{0}$, the worst point $\mathcal{F}_{N}$ and the dispersion circle $M_{G}$ of the uncertain system for $N=3$ is depicted in Fig. 2.

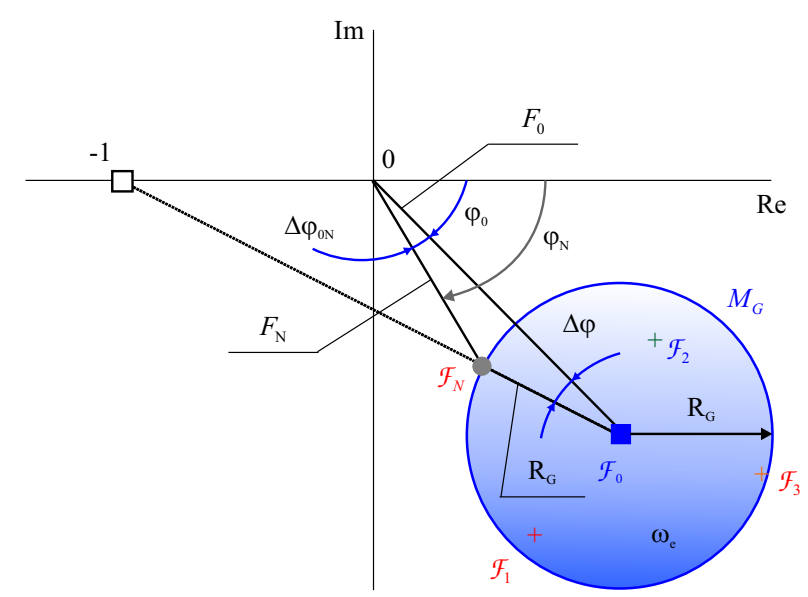

Fig. 2. Position of the nominal point $\mathcal{F}_{0}$, the "worst" point $\mathcal{F}_{n}$ and the dispersion circle $M_{G}$ of the uncertain system in the complex plane $(n=3)$ 

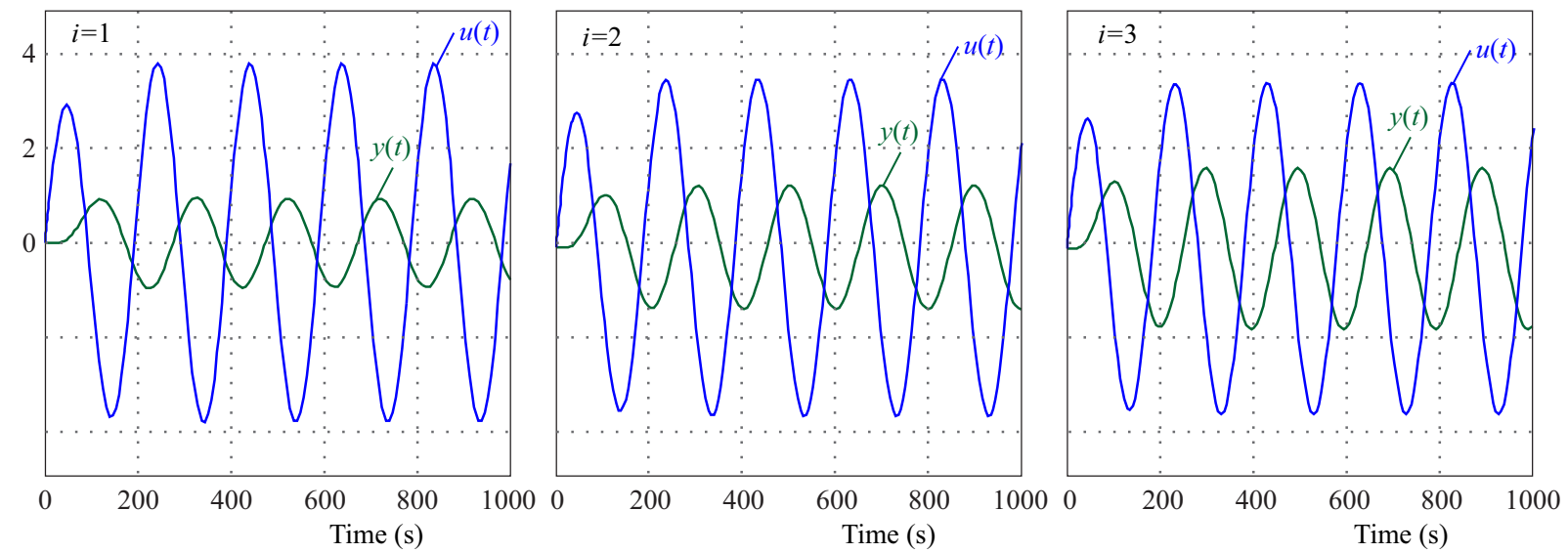

Fig. 3. Identification process of the Nyquist plots $\mathcal{F}_{i}$ for $i=1,2,3$ at the excitation frequency $\omega_{e}$

Derivation of (3)-(8) can be found in [30]. Time responses $u(t)$ and $y(t)$ during the identification stage of the uncertain plant for $N=3$ is depicted in Fig. 3 .

\section{Design of robust PID controller for non-minimum phase plants}

Following the harmonic excitation-based identification of the unknown process with uncertainties, a robust PID controller for the nominal system will be designed in this section. Turn the switch $S W$ in the control loop in Fig. 1 into the position $S W=1$. The nominal closed-loop characteristic equation

$$
C_{0}(s)=1+L_{0} s=1+F_{0}(s) F_{R}(s)=0
$$

can be divided to the following two conditions

$$
\begin{aligned}
& F_{0}\left(\omega_{e}\right) F_{R}\left(\omega_{e}\right)=\frac{1}{G_{M}}, \\
& \varphi_{0}\left(\omega_{e}\right)+\theta_{0}\left(\omega_{e}\right)=-\pi,
\end{aligned}
$$

where $G_{M}$ is the required gain margin, $\mathcal{L}_{0}(j \omega)$ is the nominal open-loop frequency-response transfer function, $\omega_{e}$ is the nominal open-loop phase crossover frequency and at the same time the excitation frequency of the controlled plant $F(s)$. Equations (10) and (11) represent the magnitude and the phase condition, respectively. Let us denote $F_{0}=\left|\mathcal{F}_{0}\left(j \omega_{e}\right)\right|, F_{R}=\left|\mathcal{F}_{R}\left(j \omega_{e}\right)\right|$ and $\varphi_{0}=\arg \mathcal{F}_{0}\left(j \omega_{e}\right)$ and $\Theta_{0}=\arg \mathcal{F}_{R}\left(j \omega_{e}\right)$, respectively. Consider the interacting PID controller in the following form

$$
F_{R}(s)=K\left[1+\frac{1}{T_{i}} s+T_{d} s\right],
$$

where $K$ is the proportional gain, $T_{i}$ is the controller integral time constant, and $T_{d}$ is controller derivative time constant, respectively. Expressing the ideal PID controller in the rectangular and polar form

$$
\mathcal{F}_{R}=K+j K\left[T_{d} \omega_{e}-\frac{1}{T_{i} \omega_{e}}\right]
$$

$$
\mathcal{F}_{R}=F_{R}\left[\cos \Theta_{0}+j \sin \Theta_{0}\right]
$$

PID coefficients can be calculated solving the complex equation at $\omega=\omega_{e}$

$$
K+j K\left[T_{d} \omega_{e}-\frac{1}{T_{i} \omega_{e}}\right]=\frac{\cos \Theta_{0}}{G_{M} F_{0}}+j \frac{\sin \Theta_{0}}{G_{M} F_{0}},
$$

where

$$
F_{R}=\frac{1}{G_{M} F_{0}}
$$

is resulting from (10). The complex equation (15) can be solved by comparing its corresponding real and imaginary parts, respectively

$$
K=\frac{\cos \Theta_{0}\left(\omega_{e}\right)}{G_{M} F_{0}\left(\omega_{e}\right)}, \quad K\left[T_{d}\left(\omega_{e}\right)-\frac{1}{T_{i}\left(\omega_{e}\right)}\right]=\frac{\sin \Theta_{0}}{G_{M} F_{0}},
$$

where the controller gain $K$ can be calculated directly from (17a). After the substitution of (17a) into (17b), a following quadratic equation in $T_{d}$ is obtained

$$
T_{d}^{2} \omega_{e}^{2}-T_{d} \omega_{e} \operatorname{tg} \Theta_{0}-\frac{1}{\mu}=0,
$$

where $\mu=T_{i} / T_{d}$ is the ratio of the integral and derivative time constant of the robust PID controller, respectively. In practical cases $\mu=4$. The controller derivative time constant $T_{d}$ can be calculated as a positive solution of (18)

$$
T_{d}=\frac{\operatorname{tg} \Theta_{0}\left(\omega_{e}\right)}{2 \omega_{e}}+\frac{1}{\omega_{e}} \sqrt{\frac{\operatorname{tg}^{2} \Theta_{0}\left(\omega_{e}\right)}{4}+\frac{1}{\mu}} .
$$

Hence, (17a) and (19) are the resulting PID tuning rules; the phase $\Theta_{0}$ can be calculated from the phase condition (11)

$$
\Theta_{0}\left(\omega_{e}\right)=-\pi-\varphi_{0}\left(\omega_{e}\right)=-\pi-\varphi_{0}\left(\omega_{e}\right) .
$$

Note that the required open-loop gain margin $G_{M}$ used in (17a) represents the tuning parameter of the designed 
Table 1. Robust PI, PD and PID controller tuning rules

\begin{tabular}{llcc}
\hline Controller & $K$ & $T_{i}$ & $T_{d}$ \\
\hline $\mathrm{PI}$ & $\frac{\cos \Theta_{0}}{G_{M} F_{0}}$ & $\frac{-1}{\omega_{e} \operatorname{tg} \Theta_{0}}$ & - \\
\hline $\mathrm{PD}$ & $\frac{\cos \Theta_{0}}{G_{M} F_{0}}$ & - & $\frac{1}{\omega_{e}} \operatorname{tg} \Theta_{0}$ \\
\hline PID & $\frac{\cos \Theta_{0}}{G_{M} F_{0}}$ & $\mu T_{d}$ & $\frac{\operatorname{tg} \Theta_{0}}{2 \omega_{e}}+\frac{1}{\omega_{e}} \sqrt{\frac{\operatorname{tg}^{2} \Theta_{0}}{4}+\frac{1}{\mu}}$ \\
\hline
\end{tabular}

Note that $F_{0}=F_{0}\left(\omega_{e}\right)$ and $\Theta_{0}=\Theta_{0}\left(\omega_{e}\right)$

PID controller to guarantee the closed-loop robust stability or performance.

Using the designed PID controller with coefficients

$$
K ; T_{i}=\mu T_{d} ; T_{d}
$$

the nominal point of the uncertain system with an unstable zero $\mathcal{F}_{0}\left(j \omega_{e}\right)$ is shifted into the phase-crossover $\mathcal{L}_{0}\left(j \omega_{e}\right)$ located on the negative real half-axis with guaranteed gain margin $G_{M}$, see Fig. 4 and 5 .

The nominal phase crossover coordinates are

$$
\left[L_{0}, \arg \mathcal{L}_{0}\left(j \omega_{e}\right)\right]=\left[1 / G_{M},-\pi\right] .
$$

The PI, PD and PID controller coefficients guaranteeing the required gain margin $G_{M}$ are obtained using the harmonic excitation-type tuning rules in Table 1 , where $\Theta_{0}=-\pi-\varphi_{0}$.

\section{Robust stability and robust performance conditions}

The designed robust PID controller has to transform the identified dispersion circle $M_{G}$, Fig. 2, centred in the nominal point $\mathcal{F}_{0}\left(j \omega_{e}\right)$ of the uncertain system into the circle $M_{L}$ centred in the nominal point $\mathcal{L}_{0}\left(j \omega_{e}\right)$ of the open-loop. Relation between the radii $R_{G}$ and $R_{L}$ of both dispersion circles $M_{G}$ a $M_{L}$ respectively is

$$
R_{L}=R_{G} F_{R}\left(\omega_{e}\right)
$$

whereby the robust stability condition has to be satisfied

$$
R_{L}<\left|1+\mathcal{L}_{0}\left(j \omega_{e}\right)\right|
$$

or robust performance condition, respectively

$$
R_{L}+R_{S}<\left|1+\mathcal{L}_{0}\left(j \omega_{e}\right)\right|
$$

Theorem 1 (Sufficient condition of robust stability under a PID controller)

Let $F(s)$ be a stable system with an unstable zero and unstructured uncertainties. Let the nominal model $F_{0}(s)$ of the controlled system be stable. Denote $\lambda_{L}=R_{L}^{+} / R_{L}$ the safety coefficient of the dispersion circle $M_{L}$. Then the closed-loop system under a PID controller is robustly stable if the following inequality holds

$$
G_{M}>1+\frac{\lambda_{L} R_{G}\left(\omega_{e}\right)}{F_{0}\left(\omega_{e}\right)}
$$

Proof:

Graphical interpretation of the PID design for robust stability as well as situation of both dispersion circles $M_{G}$ and $M_{L}$ are depicted in Fig. 4.

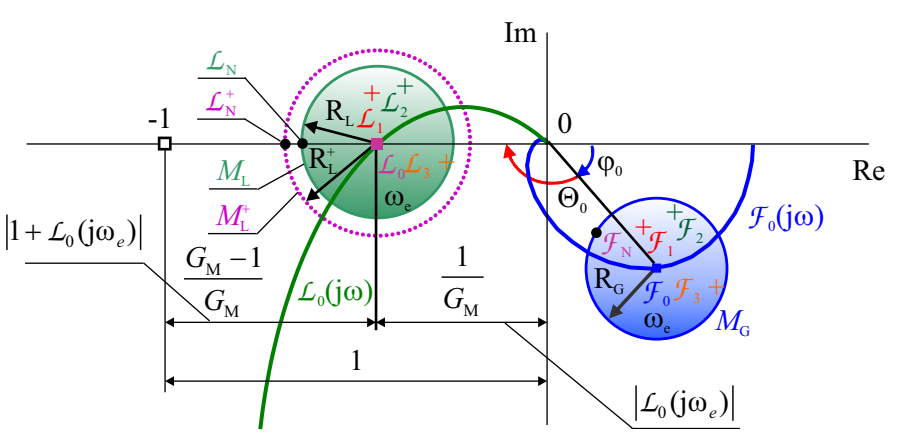

Fig. 4. Dispersion circles $M_{G}$ and $M_{L}$ for the robust stability condition

According to the Nyquist stability condition the closed loop is stable if the distance between the point $(-1,0)$ and the nominal point $\mathcal{L}_{0}\left(j \omega_{e}\right)$ is greater than the radius $R_{L}$ of the open-loop dispersion circle $M_{L}$, ie when (22) is satisfied. As illustrated in Fig. 4, the distances $\mid 1+$ $\mathcal{L}_{0}\left(j \omega_{e}\right) \mid$ and $\left|0, \mathcal{L}_{0}\right|=\left|\mathcal{L}_{0}\right|$ are related according to

$$
\left|L_{0}\right|+\left|1+\mathcal{L}_{0}\left(j \omega_{e}\right)\right|=1,
$$

which yields

$$
\left|1+\mathcal{L}_{0}\left(j \omega_{e}\right)\right|=1-L_{0}=1-\frac{1}{G_{M}} .
$$

From the gain condition (10) results the following equation

$$
L_{0}=F_{0} F_{R}=\frac{1}{G_{M}} .
$$


After substituting for the radius $R_{L}$, and carrying out several manipulations the resulting relation between the radii of dispersion circles $M_{L}$ a $M_{G}$ is obtained in the following form

$$
R_{L}=R_{G} \frac{1}{G_{M} F_{0}}
$$

Substituting (26) and (28) into the general robust stability condition (22) and considering the safety coefficient $\lambda_{L}$ we obtain the following inequality

$$
\frac{G_{M}-1}{G_{M}}>\frac{\lambda_{L} R_{G}}{G_{M} F_{0}}
$$

which can be manipulated to obtain the inequality (24) as had to be proved. In practical cases $\lambda_{L}=1.2$, and the gain margin $G_{M}$ is chosen to satisfy inequality (24). After substituting it into (17a) we can calculate coefficients of the robust PID controller.

Theorem 2 (Sufficient condition for robust performance under a PID controller)

Let $F(s)$ be a stable uncertain system described by a nominal model $F_{0}(s)$ with an unstable zero and unstructured uncertainty. Denote $\lambda_{L}=R_{L}^{+} / R_{L}$ the safety coefficient of the dispersion circle $M_{L}$. Then, robust closedloop performance under a PID controller is guaranteed if the following inequality is satisfied

$$
G_{M}>\frac{1+\lambda_{L} \frac{R_{G}}{F_{0}}}{1-\lambda_{S} \frac{G_{S}-1}{G_{S}}},
$$

where $\lambda_{S}=R_{S}^{+} / R_{S}$ is a safety coefficient of radii of the dispersion circle $M_{S}$ [30]. The prohibited area MS is defined in terms of $G_{M}$ using the expression

$$
G_{S}=\frac{1}{1-R_{S}}
$$

where $R_{S}$ is the radius of the circle $M_{S}$.

Proof: Graphical interpretation of PID design for robust performance, situation of dispersion circles $M_{G}, M_{L}$ and the prohibited area $M_{S}$ are shown Fig. 5 .

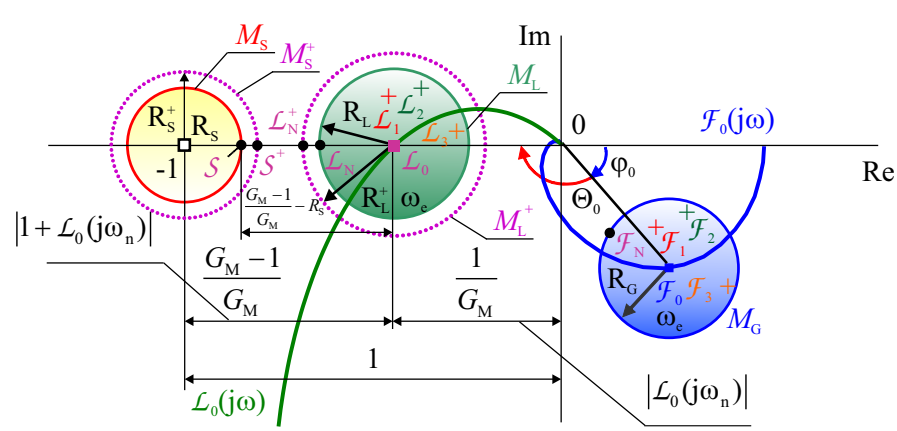

Fig. 5. Dispersion circles $M_{G}$ and $M_{L}$ and the prohibited area delineated by the circle $M_{S}$ for the robust performance condition
After substituting the distance $\left|1+\mathcal{L}_{0}\left(j \omega_{e}\right)\right|,(26)$ and the radius $R_{L},(28)$ into (23) which expresses the general robust performance condition and considering the safety coefficients $\lambda_{L}$ and $\lambda_{S}$, respectively, we obtain the following relation

$$
\frac{G_{M}-1}{G_{M}}>\frac{\lambda_{L} R_{G}}{G_{M} F_{0}}+\lambda_{S} R_{S}
$$

which after small manipulations yields the resulting relation (30).

In practical cases $\lambda_{S}=1.1$.

\section{Closed-loop performance under the designed PID controller}

Recommended frequency of the excitation signal $\omega_{e}$ generated by the sinusoid generator in the control loop in Fig.1 ranges within the following interval [31]

$$
\omega_{e} / \omega_{u} \in\langle 0.5,1.25\rangle \text { in } \mathrm{dB}
$$

where $\omega_{u}$ is the ultimate frequency of the unknown uncertain system with an unstable zero. Its value can be determined by switching $S W$ into position 3 and realizing the experiment according to Rotach [33].

In this chapter we aim to find a relation between robust performance requirements specified in the time domain in terms of $\left(t_{\mathrm{reg}}, \eta_{\max }\right)$, and PID controller parameters and frequency domain identification results in terms of $\left.G_{M}, \omega_{e}\right)$ guaranteeing their fulfilment.

Consider the interval of typical open-loop gain margins

$$
G_{M} \in\langle 3,17 \mathrm{~dB}\rangle,
$$

Let us divide the intervals (33) and (34) into linear sections $\Delta \omega_{e}=0.15 \omega_{u}$ and $\Delta G_{M}=2 \mathrm{~dB}$, respectively

$$
\begin{aligned}
& \left\{\omega_{e k} / \omega_{u}\right\}=\{0.5 ; 0.65 ; 0.8 ; 0.95 ; 1.1 ; 1.25\}, k=1 \ldots 6 \\
& \left\{G_{M j}\right\}=\{3,5,7,9,11,13,15,17\} \text { in } \mathrm{dB}, \quad j=1 \ldots 8 .
\end{aligned}
$$

In the next development, each element of the Cartesian product $\omega_{e k} \times G_{M_{j}}$ has been used to generate parameters for PID controller designed for the following benchmark examples:

$$
\begin{gathered}
F_{1}(s)=\frac{\left(1-\beta_{1} s\right)^{n_{1}}}{1+T_{1} s} \\
F_{2}(s)=\frac{\left(1-\beta_{2} s\right.}{(1+s)\left(1+T_{2} s\right)\left(1+T_{2}^{2} s\right)\left(1+T_{2}^{3} s\right)} .
\end{gathered}
$$

Closed-loop step response shaping based on using various $G_{M}$ and $\omega_{e}$ to tune PID for $F_{2}(s)$ is shown in Fig. 6 where $T_{2}=0.75$ and $\beta_{2}=1.3$; PID controllers were designed for four different gain margin values $G_{M}=$ $5,9,11,13 \mathrm{~dB}$, and three different identification levels $\omega_{e 1} / \omega_{u}=0.5, \omega_{e 3} / \omega_{u}=0.8$ and $\omega_{e 5} / \omega_{u}=1.1$. 

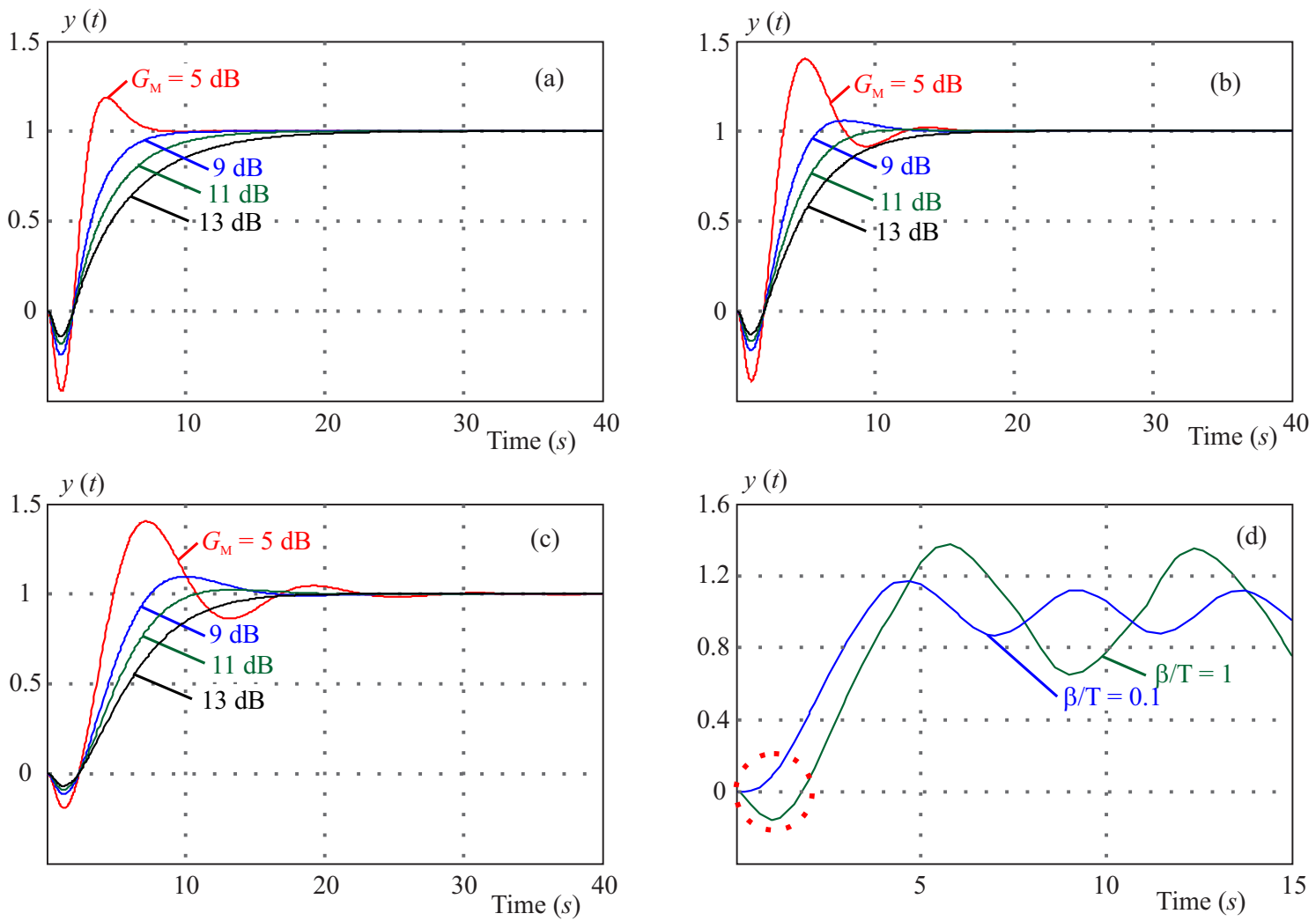

Fig. 6. Closed-loop step response shaping for (a) to (c) $-F_{2}(s)$ with $T_{2}=0.75, \alpha_{2}=1.3$ using different $G_{M}$, and (d) - relay-test time response of $F_{2}(s)$ for $\beta / T=1$ and $\beta / T=0.1$, respectively

A systematic PID design using parameters from (33) and (34) allows to evaluate and generalize the achieved performance, and to establish relation between the timedomain performance measures and the frequency domain parameters entering in PID tuning procedure

$$
\mathcal{P}:\left(\eta_{\text {max }} ; t_{\text {reg }}\right) \rightarrow\left(\omega_{e} ; G_{M}\right)
$$

Based on the fact that the designed PID controller shifts the idenitified nominal point $\mathcal{F}_{0}\left(j \omega_{e}\right)$ into the gain crossover $\mathcal{L}_{0}\left(j \omega_{e}\right)$ at the excitation frequency $\omega_{e}$, the settling time can be expressed by the relation

$$
t_{\text {reg }}=\frac{\pi \gamma}{\omega_{e}}
$$

where $\gamma$ is a curve factor of the step response. In the similar relation according to Reinisch [29] valid just for second order closed-loops its value ranges within the interval $(1 ; 4)$. In the proposed method $\gamma$ changes within a considerably broader interval $(0.5 ; 16)$ found empirically, and strongly depends on the gain margin at the given frequency $\omega_{e}$, ie $\gamma=f\left(G_{M}\right)$.

Graphical interpretation of relation (39) for different excitation frequencies $\omega_{e}$ is in Fig. 7(a) and Fig. 8(a). From the two plots clearly results that for each identification level $\omega_{e k} / \omega_{u}$ with increasing the open-loop gain margin $G_{M}$ the settling time $\tau_{\text {reg }}$ first decreases and after achieving its minimum value $\tau_{\text {reg min }}$ it grows again.

Consider the benchmark examples (36) and (37) with the following parameter values $F_{1.1}(s):\left(T_{1 n_{1}}, \beta_{1}\right)=$
$(0.75,8,0.2) ; F_{1.2}(s):(1,3,0.1) ; F_{1.3}(s):(0.5,5,1) ;$ $F_{2}(s): T_{2}=0.5, \beta_{2}=1.3$.

The couples of studied systems $\left[F_{2}(s), F_{1.3}(s)\right]$ and $\left[F_{1.2}(s), F_{1.1}(s)\right]$ principally differ by the ratio $\beta / T$; for the first couple $\left[\beta_{2} / T_{2}=2.6, \beta_{1.3} / T_{1.3}=2\right]$, and for the second one $\left[\beta_{1.2} / T_{1.2}=0.1, \beta_{1.1} / T_{1.1}=0.27\right]$.

Thus, for the performance of a closed-loop comprising a non-minimum phase system with an unstable zero and a PID controller, the ratio of the parameter $\beta$ and the (dominant) time constant is decisive. Based on analysis realized for a large number of benchmark examples [34] it has been concluded that unknown controlled systems with an unstable zero can be classified with respect to the ratio $\beta / T$ into the following two groups:

1. systems with an unstable zero and $\beta / T<0.3$

2 . systems with an unstable zero and $\beta / T>0.3$

For nonminimum-phase systems with an unstable zero the B-parabolas for $\eta_{\max }$ depending on different gain margins $G_{M}$ and excitation levels $\omega_{e} / \omega_{u}$ are shown in Fig. 7(b) (for $\beta / T>0.3$ ) and in Fig. 8(b) (for $\beta / T<$ $0.3)$. We can observe that increasing the gain margin $G_{M}$ brings about decreased maximum overshoot $\eta_{\max }$.

\section{Discussion}

- Note that the ratio $\beta / T$ cannot be found mathematically because the plant model is unknown. To decide to which category the controlled plant belongs $(\beta / T>0.3$ or $\beta / T<0.3)$ it is sufficient to analyze 

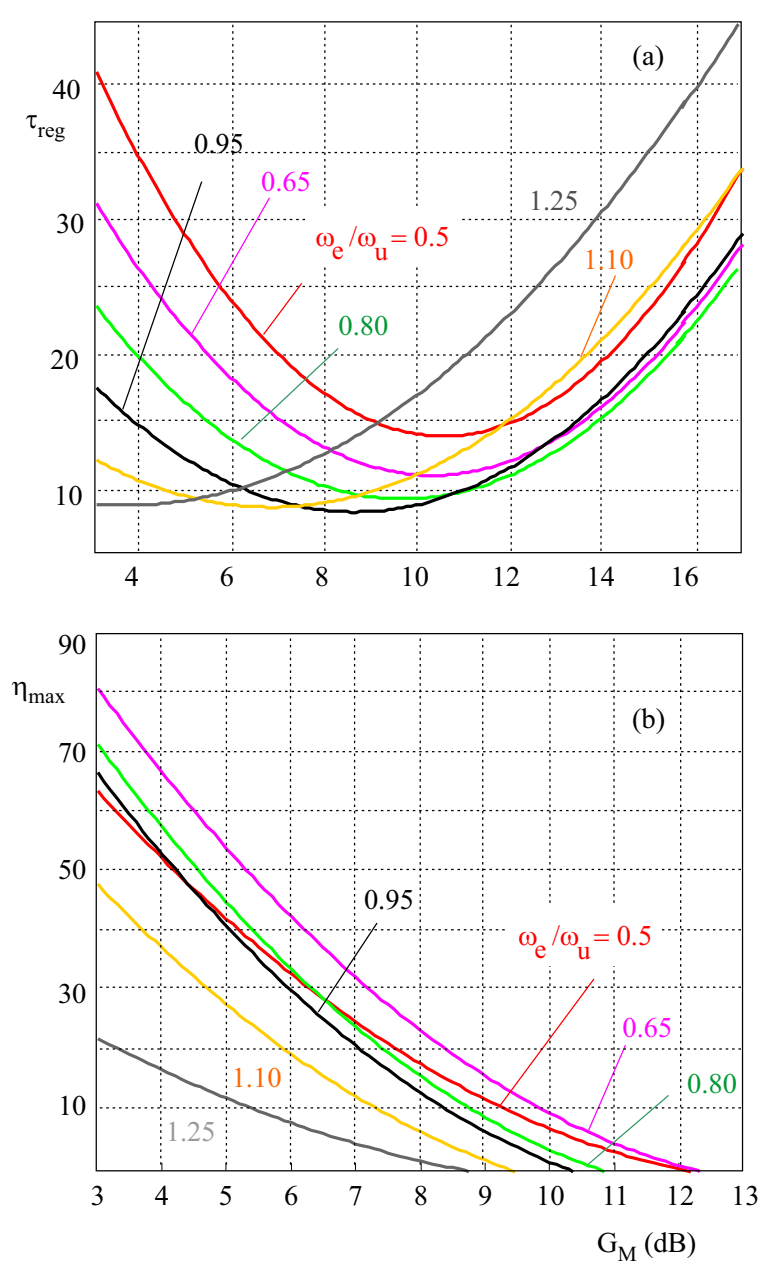

Fig. 7. Dependences: (a) $-\tau_{\text {reg }}=\omega_{u} t_{\text {reg }}=f\left(G_{M} ; \omega_{e}\right)$, (b) $\eta_{\max }=f\left(G_{M} ; \omega_{e}\right) ;$ for various identification levels $\omega_{e} / \omega_{u}$ $k=1 \ldots 6, \beta / T>0.3$

the beginning of the rising part of the output variable during the Rotach test for determination of the critical frequency $\omega_{u}$. If the $y(t)$ response is S-shaped with just a tiny undershoot under the time axis, the controlled object belongs to the category $\beta / T<0.3$ and B-parabolas in Fig. 8 are to be used. However, if a considerable square root-shaped undershoot of $y(t)$ under the time axis appears, Fig. 6(d), in the red dashed ellipse, the system falls into the category $\beta / T>0.3$ and its performance will be assessed using the B-parabolas in Fig. 7.

- B-parabolas for $\beta / T>0.3$ in Fig. 7(a) indicate zero overshoot at gain margins greater than $12.5 \mathrm{~dB}$. When tuning a PID controller using $G_{M}$ larger than $12.5 \mathrm{~dB}$ the settling time rises almost linearly for all identification levels $\omega_{e} / \omega_{u}$, Fig. $7($ b). The range from 12.5 $\mathrm{dB}$ to $17 \mathrm{~dB}$ can be used to purposefully increase the settling time in case that a larger rise time of the step response is required ( $e g$ on increasing pressure in a system where a sudden increase may cause a pipe breakage).

- B-parabolas for $\beta / T<0.3$ in Fig. 8(a) clearly show that to achieve $\eta_{\max }=0 \%$ it is necessary to use a
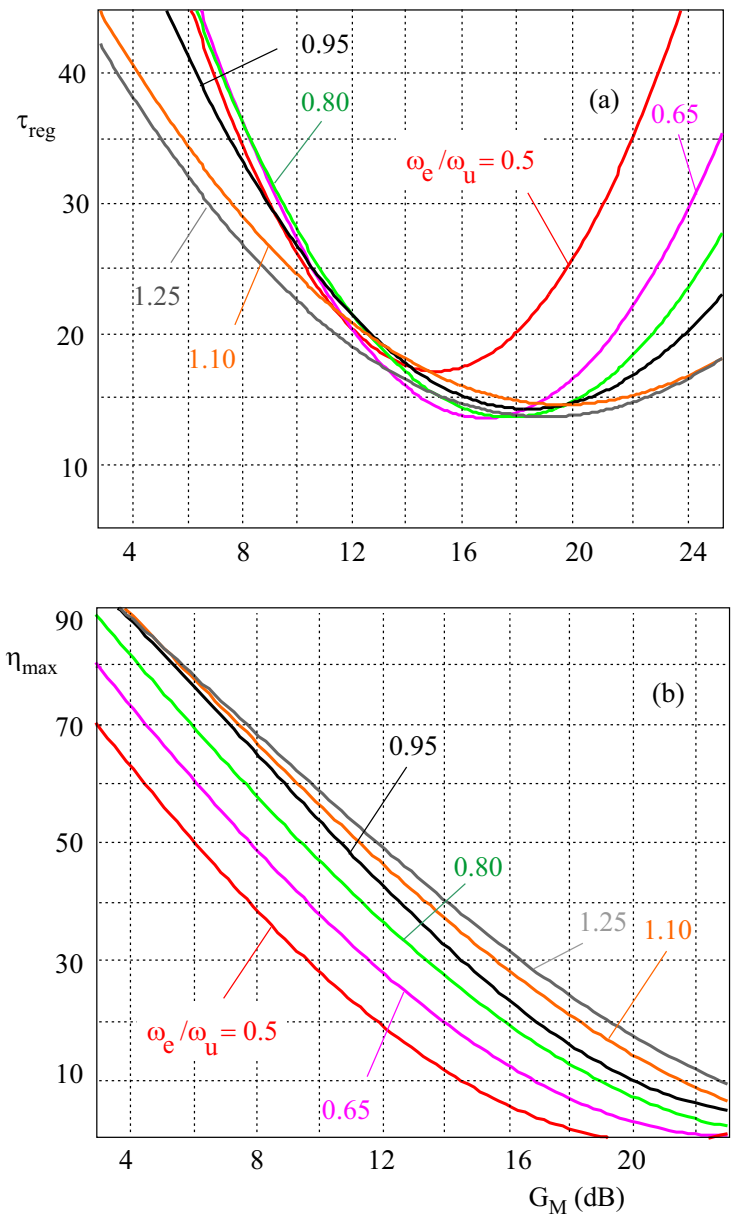

Fig. 8. Dependences: (a) $-\tau_{\text {reg }}=\omega_{u} t_{\text {reg }}=f\left(G_{M} ; \omega_{e}\right)$, (b) $\eta_{\max }=f\left(G_{M} ; \omega_{e}\right)$; for various identification levels $\omega_{e} / \omega_{u}$, $k=1 \ldots 6, \beta / T<0.3$

gain margin larger by approximately $10 \mathrm{~dB}$ compared with systems with the ratio $\beta / T>0.3$. The shortest achievable relative settling time for systems with $\beta / T<0.3$ is $\tau_{\text {reg }}=13$ according to B-parabolas in Fig. 8(b). The smallest overshoot for the given gain margin can be achieved at the lowest identification level $\omega_{e} / \omega_{u}=0.5$ (red parabola).

- With increasing the gain margin the maximum overshoot of the closed-loop step response decreases. For a chosen gain margin, it is possible to reduce the settling time by increasing frequency of the sinusoid signal, however at the price of increasing the step response undershoot under the time axis (Fig. 6).

The robust PID controller is designed using the harmonic excitation-based design method described in chapters 2 and 3 ; the input data for the nominal model $\mathcal{F}_{0}\left(j \omega_{e}\right)$ are its coordinates: $\left\{F_{0}\left(\omega_{e}\right) ; \varphi_{0}=\arg \mathcal{F}_{0}\left(j \omega_{e}\right)\right\}$. It is a well known fact from control theory that the openloop gain margin $G_{M}$ is an attractive indicator of robustness [2] and at the same time the design parameter of the PID controller gain (17a). Its value has to be chosen from the B-parabolas according to the prescribed closed-loop 
performance specification fulfilling the robust stability or robust performance condition.

Determination of the gain margin $G_{M}$ for nominal performance and robust stability

When calculating coefficients of the robust PID controller according to Tab. 1, the value of the gain margin $G_{M}$ has to be chosen from the couple of B-parabolas in Fig. 7 or Fig. 8 to guarantee nominal performance (ie not exceeding specified $\eta_{\max 0}$ and $t_{\text {reg0 }}$ by the nominal model closed-loop); and simultaneously meeting the derived robust stability condition (24).

Determination of the gain margin $G_{M}$ for robust performance and robust stability

The value of the robust performance magnitude $G_{S}$, (32) is determined using the B-parabolas (Fig. 7 or Fig. 8) so as to guarantee the specified $\eta_{\max N}$ and $t_{\operatorname{reg} N}$ for the "worst" identified point of the plant; by substituting it into (34) we obtain the radius $R_{S}$ subsequently used in the robust performance condition (31) to calculate the gain margin $G_{M}$; coefficient of the PID controller are then calculated according to Tab. 1.

Verification of the proposed harmonic excitation-based robust PID controller design method is illustrated on the following examples.

\section{Verification}

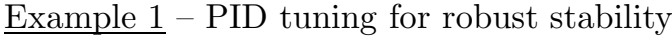

Consider the following uncertain plant F3(s) with an unstable zero

$$
\begin{gathered}
F_{3}(s)=\frac{K_{3}\left(1-\beta_{3} s\right)}{\left(1+T_{3} s\right)^{3}} \\
F_{30}(s)=\frac{K_{30}\left(1-\beta_{30} s\right)}{\left(1+T_{30} s\right)^{3}}=\frac{0.8(1-7.5 s)}{(1+27.5 s)^{3}}
\end{gathered}
$$

with parameters $K_{3}, T_{3}$ and $\beta_{3}$ varying within $\pm 15 \%$ around the nominal values; $F_{30}(s)$ is the nominal model. Let us design a robust PID controller to guarantee a maximum relative settling time $\tau_{s 0}=12$ and a maximum overshoot $\eta_{\max 0}=5 \%$ for the nominal model $F_{30}(s)$, and stability of the whole family of the uncertain plants $F_{30}(s)$ with unstable zero (ie robust stability).

\section{Solution and discussion}

1. Measured ultimate frequency of the nominal model was $\omega_{u}=0.0488 \mathrm{rad} / \mathrm{s}$. From the required nominal closed-loop performance results $t_{\text {reg }}=\tau_{\text {reg }} / \omega_{u}=245.9 \mathrm{~s}$.

2 . The expected nominal performance $\left(\eta_{\max 0}, \tau_{\text {reg0 } 0}\right)$ $=(5 \%, 12)$ is achievable for $\left.\left(G_{M}, \omega_{e}\right)=18 \mathrm{~dB}, 0.65 \omega_{u}\right)$ based on "pink" B-parabolas in Fig. 8, $\beta_{30} / T_{30}<0.3$. As there are three uncertain parameters in $F_{3}(s): K_{3}, T_{3}$ and $\beta_{3}$, the number of identifications is $N=2^{3}$.

3. Using the excitation frequency $\omega_{e}=0.65 \omega_{u}=0.0317$ $\mathrm{rad} / \mathrm{s}$ eight points of the Nyquist plots of the uncertain plant $\mathcal{F}_{31}\left(j \omega_{e}\right) \ldots F_{38}\left(j \omega_{e}\right)$ were identified using the sinusoid excitation method (blue $\times$ in Fig. 9). The nominal point $F_{30}\left(j \omega_{e}\right)$ obtained from coordinates of the identified points $\mathcal{F}_{3 i}\left(j \omega_{e}\right), i=1 \ldots 8$ is located on the Nyquist plot of the nominal model (blue) proving correctness of the identification. The dispersion circle $M_{G}$ is centred in the nominal point $\mathcal{F}_{30}\left(j \omega_{e}\right)$ and its radius is $R_{G}=0.164$.

4. As $G_{M}=18 \mathrm{~dB}$ and the right-hand side of (24) is $G_{\mathrm{RS}}=3.52 \mathrm{~dB}$, the robust performance condition (24) $G_{M}>G_{\mathrm{RS}}$ is satisfied. The designed robust PID controller shifts the nominal point $\mathcal{F}_{30}\left(j \omega_{e}\right)$ of the controlled system to the negative half-axis of the complex plane into the point $\mathcal{L}_{30}\left(j \omega_{e}\right)=\mathcal{F}_{30}\left(j \omega_{e}\right) \mathcal{F}_{R \text { rob }}\left(j \omega_{e}\right)=0.12 e^{-j \pi}$, which is a point of the nominal open-loop Nyquist plot $\mathcal{L}_{30}\left(j \omega_{e}\right)$ (green plot in Fig. 9). Thus, the gain margin $G_{M}=18 \mathrm{~dB}$ for the nominal closed-loop is guaranteed. Closed-loop step response with the nominal model (green plot in Fig. 10) verifies achieving the required nominal performance $\eta_{\max 0 \mathrm{obt}}=4.55 \%, \tau_{\text {reg0obt }}=\omega_{u} t_{\mathrm{reg} 0}$ $=11.86$.

5. The dispersion circle $M_{\text {Lobt }}$ (green) in Fig. 10 with the radius $R_{L}=0.0573$ encompasses all points $\mathcal{L}_{3 i}\left(j \omega_{e}\right)=\mathcal{F}_{3 i}\left(j \omega_{e}\right) \mathcal{F}_{R \text { rob }}\left(j \omega_{e}\right)$ for $i=1 \ldots 8$. Using the PID controller, the worst point of the robust stability controlled system $\mathcal{F}_{3 N}\left(j \omega_{e}\right)$ was shifted to $\mathcal{L}_{3 N}\left(j \omega_{e}\right)=$ $0.16 e^{-j 1.09 \pi}$ yielding the estimated worst open-loop gain $\operatorname{margin} G_{M N}=14.9 \mathrm{~dB}$.

6. The smallest gain margin $G_{M N}^{+}=13.1 \mathrm{~dB}$ with the corresponding worst point $\mathcal{F}_{3 N}\left(j \omega_{e 0}\right)$ given by the intersection of the red open-loop Nyquist plot and the negative real half-axis of the complex plane. According to the "pink" B-parabolas in Fig. 8, under the identification level $\omega_{e} / \omega_{u}=0.65$ the expected maximum overshoot is $\eta_{\max N}=25 \%$ and the relative settling time $\tau_{\text {reg } N}=16$. The closed-loop step response in Fig. 10 (red plot) verifies that the achieved performance measure values $\eta_{\max N}=$ $13.5 \%, t_{\mathrm{reg} N}=301 \mathrm{~s}$ correspond with the required ones.

\section{$\underline{\text { Example } 2}$ - PID tuning for robust performance}

Consider again the uncertain plant $\mathcal{F}_{3}(s)$ from Example 1 , with same uncertain parameters $K_{3}, T_{3}$ and $\beta_{3}$. Let us design a robust PID controller to guarantee a relative settling time $\tau_{\operatorname{reg} N}=12$ and maximum overshoot $\eta_{\max N}=5 \%$ for all models from the family of plants $F_{3}(s)$ (ie robust performance).

Solution and discussion

1. The task can be solved using the robust performance magnitude $G_{S}=18 \mathrm{~dB}$ and identification level $\omega_{e} / \omega_{u}=$ 0.65 resulting from the "pink" B-parabolas in Fig. 8.

2. The calculated right-hand side of $(31)$ is $G_{0 R P}=$ $21.37 \mathrm{~dB}$, hence the robust performance condition (30) $G_{M}>G_{0 R P}$ is fulfilled if the gain margin is chosen eg $G_{M}=21.5 \mathrm{~dB}$.

3. Using the designed robust PID controller, the point $\mathcal{F}_{30}\left(j \omega_{e}\right)$ of the nominal plant is moved on the unit circle into the point $\mathcal{L}_{30}\left(j \omega_{e}\right)=\mathcal{F}_{30}\left(j \omega_{e}\right) \mathcal{F}_{R \text { rob }}\left(j \omega_{e}\right)=$ $0.0841 e^{-j \pi}$. The nominal open-loop Nyquist plot (green 


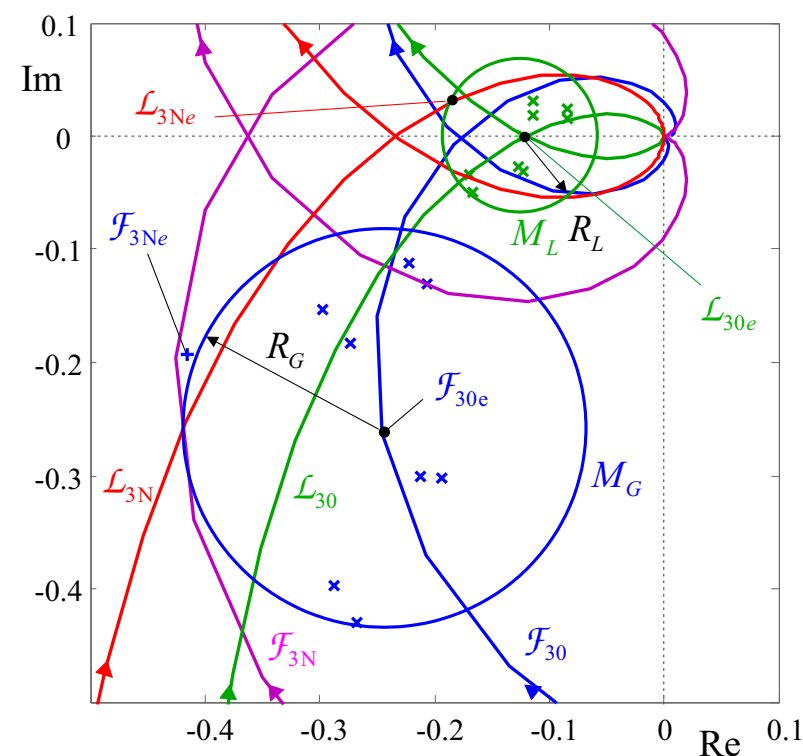

Fig. 9. Nyquist plots of plants $\mathcal{F}_{30}(j \omega), \mathcal{F}_{3 N}(j \omega)$ and openloops $\mathcal{L}_{30}(j \omega), \mathcal{L}_{3 N}(j \omega)$ with PID controllers designed for $\left(\tau_{\text {reg } 0} ;\right.$ $\left.\eta_{\max 0}\right)=(12 ; 5 \%)$, robust stability. Points with indices containing letter " $\mathrm{e}$ " pertain to the excitation frequency.

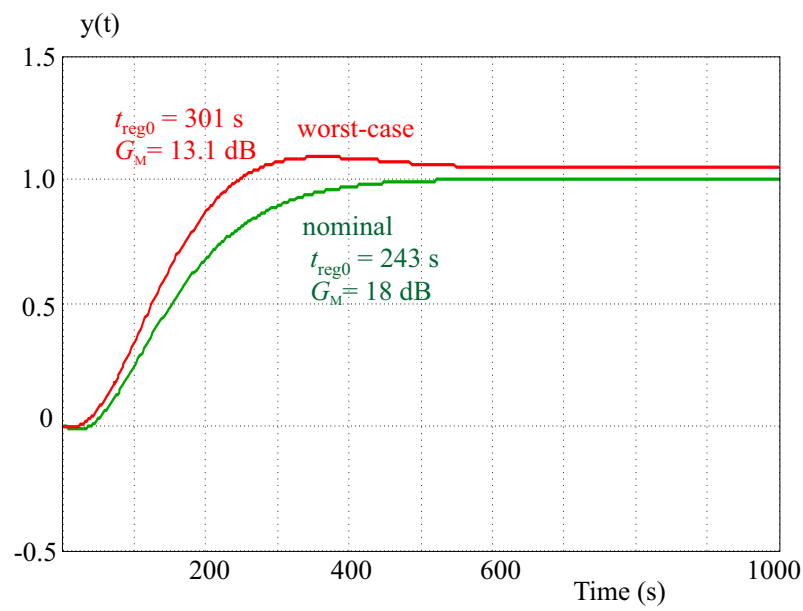

Fig. 10. Robust stability-guaranteed closed-loop step responses $y(t)$ with robust PID controller designed for $\left(\tau_{\operatorname{reg} 0} ; \eta_{\max 0}\right)=$ $(12 ; 5 \%)$

Table 2. Desired and obtained performance measures under the designed robust PID controllers designed for the uncertain plant $F_{3}(s)$

\begin{tabular}{lcccc}
\hline Design for: & $\mathrm{NP}+\mathrm{RS}$ & $(\mathrm{dB})$ & $\mathrm{NP}+\mathrm{RP}$ & $(\mathrm{dB})$ \\
\hline$\eta_{\text {max0 des }}$ & $5 \%$ & $G_{M 0}=18.0$ & - & $G_{M}==22$ \\
$t_{\text {reg0 des }}$ & $245.9 \mathrm{~s}$ & & - & \\
\hline$\eta_{\max N \text { des }}$ & - & $G_{M N}^{+}=14.9$ & $5 \%$ & $G_{M N}^{+}=18.8$ \\
$t_{\text {reg } N \text { des }}$ & - & & $245.9 \mathrm{~s}$ & \\
\hline$\eta_{\max 0 \mathrm{obt}}$ & $4.5 \%$ & $G_{M 0}=18.0$ & $0 \%$ & $G_{M 0}=22.0$ \\
$t_{\text {reg0obt }}$ & $243 \mathrm{~s}$ & & $381 \mathrm{~s}$ & \\
\hline$\eta_{\max N \text { obt }}$ & $13.5 \%$ & $G_{M N}^{+}=13.1$ & $4.8 \%$ & \multirow{2}{*}{$G_{M N}^{+}=16.9$} \\
$t_{\text {reg } N \text { obt }}$ & $301 \mathrm{~s}$ & & $237 \mathrm{~s}$ & \\
\hline
\end{tabular}

Abbreviations: NP - (Nominal Performance)

RS - (Robust Stability), RP - (Robust Performance)

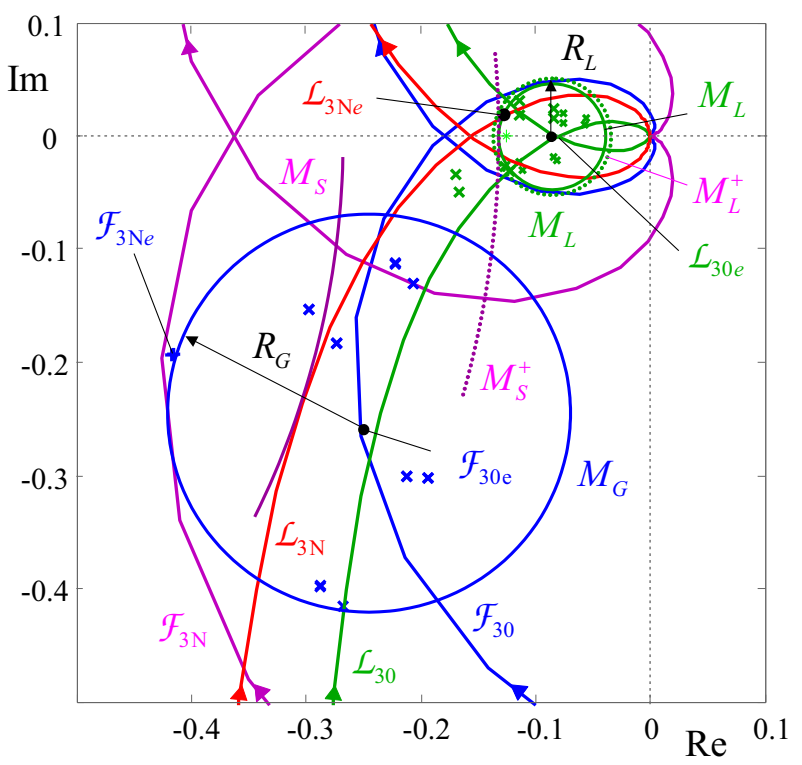

Fig. 11. Nyquist plots of plants $\mathcal{F}_{30}(j \omega), \mathcal{F}_{3 N}(j \omega)$ and openloops $\mathcal{L}_{30}(j \omega), \mathcal{L}_{3 N}(j \omega)$ with PID controllers designed for $\left(\tau_{\text {reg } 0} ;\right.$ $\left.\eta_{\max 0}\right)=(12 ; 5 \%)$, robust performance. Points with indices containing letter "e" pertain to the excitation frequency.

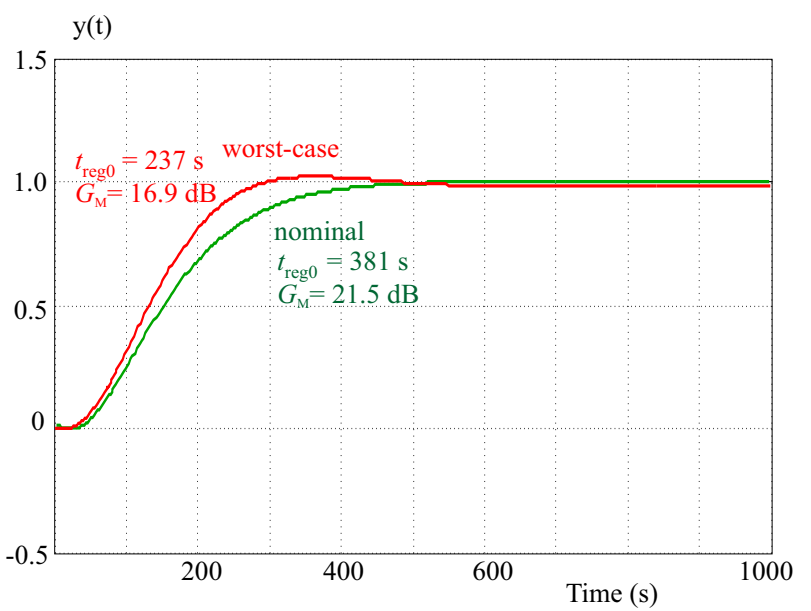

Fig. 12. Robust performance-guaranteed closed-loop step responses $y(t)$ with robust PID controller designed for $\left(\tau_{\operatorname{reg} N} ; \eta_{\max N}\right.$ $=(12 ; 5 \%)$

Table 3. Locations of the points $\mathcal{F}_{30}, \mathcal{L}_{30}, \mathcal{F}_{3 N}, \mathcal{L}_{3 N}$, radii of the dispersion circles $R_{G}, R_{L}, R_{S}$ and coefficients of the robust PID controllers designed for $F_{3}(s)$

\begin{tabular}{lcc}
\hline $\mathcal{F}_{30}\left(j \omega_{e 0}\right)$ & \multicolumn{2}{c}{$0.36 e^{-j 0.76 \pi}$} \\
$\mathcal{F}_{3 N}\left(j \omega_{e 0}\right)$ & \multicolumn{2}{c}{$0.46 e^{-j 0.86 \pi}$} \\
$R_{G}$ & \multicolumn{2}{c}{0.164} \\
$\mathcal{F}_{R}\left(j \omega_{e}\right)$ & $0.35 e^{-j 0.24 \pi}$ & $0.23 e^{-j 0.23 \pi}$ \\
$K_{\text {rob }}$ & 0.258 & 0.173 \\
$T_{i \text { rob }}$ & 27.729 & 27.729 \\
$T_{d \text { rob }}$ & 6.932 & 6.932 \\
$\mathcal{L}_{30}\left(j \omega_{e 0}\right)$ & $0.12 e^{-j \pi}$ & $0.08 e^{-j \pi}$ \\
$\mathcal{L}_{3 N}\left(j \omega_{e 0}\right)$ & $0.16 e^{-j 1.1 \pi}$ & $0.11 e^{-j 1.1 \pi}$ \\
$R_{L}$ & 0.0573 & 0.0400 \\
$R_{S}$ & 0.7228 & 0.6992 \\
\hline
\end{tabular}


curve) passes through the point $\mathcal{L}_{30}\left(j \omega_{e}\right)$, Fig. 11. Compared with the design task from Example 1, the circle $M_{L}$ has moved towards the imaginary axis of the complex plane, and its radius has reduced to $R_{L}=0.0400$ (in the Example 1 was $R_{L}=0.0573$ according to Fig. 9).

4. The lowest phase margin estimated from the position of the "worst" point $\mathcal{L}_{3 N}\left(j \omega_{e 0}\right)=0.112 e^{-j 1.1 \pi}$ is $G_{M N}^{+}=18.8 \mathrm{~dB}$. Intersection of the red Nyquist plot with the real negative half-axis of the complex plane yields the actual achieved lowest open-loop phase margin $G_{M N}^{+}=16.9 \mathrm{~dB}$.

5. Radius of the prohibited circle is $R_{S}=\left(G_{S}-\right.$ $1) / G_{S}=0.699$; its value multiplied by the expansion coefficient $\lambda_{S}=1.2$, and increased radius $R_{L}$ of the dispersion circle $M_{L} \lambda_{L}=1.1$ times guarantee that no openloop Nyquist plot enters the prohibited area delimited by the circle $M_{S}$. The expanded circles $M_{L}^{+}$a $M_{S}^{+}$in Fig. 11 (dotted curves) are touching which indicates fulfilment of the robust performance condition (30) as well as the generalized robust performance condition (23).

6. Closed-loop step response of the "worst" plant model (Fig. 12, red curve) shows $\eta_{\max N \text { obt }}=4.8 \%$ and a relative settling time $\tau_{\text {reg } N \text { obt }}=\omega_{u} t_{\text {reg } N \text { obt }}=11.57$, which proves fulfilment of the input performance requirements. PID controller has been designed for the nominal model of the plant using the gain margin $G_{M}=21.5$ $\mathrm{dB}$ and the identification level $\omega_{e}=0.65 \omega_{u}$, respectively, according to the pink parabolas showed in Fig. 8, hence the expected nominal closed-loop performance is $\eta_{\max 0}=1.5 \%$ and $\tau_{\text {reg0 }}=21$. The nominal closed-loop step response depicted in Fig. 12 (green curve) shows the obtained performance values $\eta_{\max 0 \mathrm{obt}}=0 \%$ and $\tau_{\text {reg obt }}=18.59$, in compliance with the expected performance.

The results achieved for the uncertain plant $F_{3}(s)$ with an unstable zero according to Examples 1 and 2 are summarized in Table 2 and 3 which includes: locations of the nominal point $\mathcal{F}_{30}\left(j \omega_{e}\right)$ and the worst-case point $\mathcal{F}_{3 N}\left(j \omega_{e}\right)$, radii of the dispersion circles $R_{G}, R_{L}$ and $R_{S}$ and coefficients of the designed robust PID controllers, required performance specified by the designer, achieved performance and gain margins used as design parameters.

\section{Conclusions}

Based on the results of Examples 1 and 2, and application of the developed harmonic excitation-based robust PID design method for a broad range of benchmark examples [34], the following conclusions can be drawn:

Increasing degree of robust stability is achieved by decreasing maximum overshoot of the nominal closedloop step response.

For a maximum change $\kappa=30 \%$ in uncertain parameters of the plant with an unstable zero it is possible to achieve $\eta_{\max } \in\langle 0,90\rangle \%$ and $t_{\mathrm{reg}} \in\left\langle 8.5 / \omega_{u}, 45 / \omega_{u}\right\rangle$ using the proposed method for both the nominal point and the worst case point $\mathcal{F}_{N}$ of the corresponding family of plants with unknown mathematical model $\left(\omega_{u 0}\right.$ is an ultimate frequency of the corresponding nominal model). Individual intervals result from the ranges of $\eta_{\max }=f\left(G_{M}, \omega_{e}\right)$ and $t_{\mathrm{reg}}=f\left(G_{M}, \omega_{e}\right)$ according to the B-parabolas in Fig. 7 and 8. The proposed method allows the designer to directly use the performance specification in terms of $\eta_{\max }$ and $t_{\text {reg }}$ in the robust controller design algorithm operating in the frequency domain for both the nominal and the worst case plant model (using the performance B-parabolas).

\section{REFERENCES}

[1] M. Vítečková, A. Smutný and L. Víteček, "Controller Tuning for Controlled Plants With Time Delay", Preprints of Proceedings of Pid'00: IFAC Workshop on Digital Control, Terrassa, Spain, Pp.283-288, 2000.

[2] K. J. Åström and T. Hägglund, PID Controllers: Theory, Design and Tuning, 2nd Edition.Instrument Soceity of America.ISBN: 1556175167, 1995.

[3] K. Cohen and D. E. Bosett, "Fuzzy Logic NonMinimum Phase Autopilot Design", AIAA Guidance, Navigation, and Control Conference and Exhibit, 11-14 August 2003, Austin, Texas, Paper 2003-5550.

[4] J. Hauser, S. Sastry and G. Meyer, "Nonlinear Control Design for Slightly NonMinimum Phase Systems: Application to V/STOL Aircraft", Automatica, vol. 28, no. 4, Pp.665-679, 1992.

[5] D. S. Kwon and W. J. Book, "A TimeDomain Inverse Dynamic Tracking Control of A SingleLink Flexible Manipulator", J. Dyn. Syst. T. ASME, vol. 116, pp.193-200, 1994.

[6] C. Kravaris and P. Daoutidis, "Nonlinear State Feedback Control of SecondOrder NonminimumPhase Nonlinear Systems", Comput. Chem. Eng., vol. 14, no. 4/5, Pp.439-449, 1990.

[7] P. R. Gray and R. G. Meyer, Analysis and Design of Analog Integrated Circuits, 3rd Ed., New York, Wiley, 1993.

[8] M. M. Seron, J. H. Braslavsky and G. C. Goodwin, Fundamental Limitations Filtering and Control, New York, Springer Verlag, 1997.

[9] M. M. Seron, J. H. Braslavsky, P. V. Kokotovic and D. Q. Mayne, "Feedback Limitations Nonlinear Systems: From Bode Integrals to Cheap Control", IEEE T. Automat. Contr., vol. 44, no. 4, Pp.829-833, April 1999.

10] L. Qiu and E. J. Davison, "Performance Limitations of Nonminimum Phase Systems The Servomechanism Problem", Automatica, vol. 29, no. 2, Pp.337-349, 1993.

[11] R. H. Middleton, "Tradeoffs Linear Control System Design", Automatica, vol. 27, no. 2, pp.281-292, 1991.

[12] J. B. Hoagg and D. S. Bernstein, "Nonminimum Phase Zeros. IEEE Contr", Syst. Mag., vol. 27, no. 3, Pp.45-57, 2007.

[13] J. C. Doyle, B. A. Francis and A. R. Tannenbaum, Feedback Control Theory, New York, MacMillan, 1992.

[14] S. Skogestad and I. Postlethwaite, Multivariable Feedback Control, New York, Wiley, 1996.

[15] T. Kailath, Linear Systems, Englewood Cliffs, Prentice Hall, 1980.

[16] A. P. Aguiar, J. P. Hespanha and P. V. Kokotovic, "Pathfollowing for Nonminimum Phase Systems Removes Performance Limitations", IEEE T. Automat. Contr., vol. 50, no. 2, Pp.234-239, 2005.

[17] M. Fliess, H. Marquez and R. Siraramírez, "Regulation of Nonminimum Phase Outputs: A Flatness Based Approach", Perspectives Control Theory and Applications: A Tribute to Ioan Doré Landau, D. Normand Cyrot, Ed. London, UK, "Springer Verlag, 1998, Pp.143-164. 
[18] S. Alhiddabi and N. Mcclamroch, "Tracking and Maneuver Regulation Control for Nonlinear Nonminimum Phase Systems: Application to Flight Control", IEEE T. Contr. Syst. T. , vol. 10, no. 6, Pp.780-792, 2002

[19] K. J. Åström and T. Hägglund, Advanced PID Control, ISA, 2005.

[20] B. S. Chen and T. Y. Yang, "Robust Optimal Model Matching Control Design for Flexible Manipulators", J. Dyn. Syst. T. ASME, vol. 115, Pp.173-178, 1993.

[21] M. T. Ho and Y. W. Tu, "PID Controller Design for A Flexible-Link Manipulator", Proceedings of The 44th IEEE Conference On Decision and Control, and The European Control Conference 2005, Seville, Spain, December 1215, 2005, Pp.6841-6846.

[22] R. P. Sree and M. Chidambaram, "Simple Method of Tuning PI Controllers for Stable Inverse Response Systems", Journal of The Indian Institute of Science, 83 (May-August), pp.73-82, 2003.

[23] Q. G. Wang, Y. Zhang and X. Guo, "Robust Closed-loop Identification with Application to Auto-tuning", Journal of Process Control, 11, Pp.519530.

[24] H. P. Huang, M. W. Lee and C. L. Chen, "Inverse-Based Design for A Modified PID Controller", Journal of The Chinese Institute of Chemical Engineers, 31, Pp.225-236, 2000.

[25] A. O'Dwyer, "A Summary of PI and PID Controller Tuning Rules for Processes with Time Delay", IFAC PID'00, Part 1, Pp.175180, Part 2, pp.242-247, 2000.

[26] É. Poulin and A. Pomerleau, "PID Tuning for Integrating and Unstable Processes", IEEE Proceedings Control Theory and Application, 143, Pp.429-435.

[27] A. O'Dwyer, "PI and PID Controller Tuning Rule Design for Processes with Delay, to Achieve Constant Gain and Phase Margins for All Values of Delay", Proceedings of The Irish Signals and Systems Conference (N. U. I. Maynooth, Ireland), pp.96100, 2000 .

[28] I. L. Chien, "IMC-PID Controller Design an Extension", Proceedings of The IFAC Adaptive Control Chemical Processes Conference, Copenhagen, Denmark, Pp.147-152, 1988.

[29] K. Reinisch, "Kybernetische Grundlegen Und Beschreibung Kontinuierlicher Systems", Veb Verlag Technik, Berlin, 1974.

[30] Š. Bucz and A. Kozáková, "PID Controller Design for Specified Performance", Introduction to PID Controllers: Theory, Tuning and Application to Frontier Areas, Department of Chemical Engineering, CLRI, Adyar, India, ISBN 9789533079271, 2012.

[31] Š. Bucz, A. Kozáková and V. Veselý, "Robust PID Controller Design for Performance Based On Ultimate Plant Parameters",
IFAC Papers online: 8th IFAC Symposium On Robust Control Design ROCOND 2015, Bratislava, Slovakia, 2015, pp. 388-395.

32] Bucz and Š, "Engineering Methods of PID Controller Tuning for Specified Performance", Doctoral Thesis, Slovak University of Technology Bratislava, Slovak Republic (In Slovak), 2011.

33] V. Rotach, "Avtomatizacija Nastrojki System Upravlenija", Energoatomizdat, Moskva, Russia (In Russian), 1984.

[34] K. J. Åström and T. Hägglund, "Benchmark Systems for PID Control", IFAC PID’00, Terrasa, pp.181-182, 2000.

Received 21 December 2017

Štefan Bucz $(\mathrm{PhD})$ was born in Komárno, Slovakia, in 1978. He graduated from the Faculty of Electrical Engineering and Information Technology, Slovak University of Technology (FEI STU) in Bratislava, in 2005 and received his $\mathrm{PhD}$ degree in Automation and Control in 2011. He is externally working in the Institute of Robotics and Cybernetics, FEI STU in Bratislava. The main field of his research is robust control, PID controller design and tuning for specified performance for industrial processes with unknown mathematical model and varying parameters.

Alena Kozáková, (Assoc Prof, $\mathrm{PhD}$ ) graduated from the Faculty of Electrical Engineering and Information Technology, Slovak University of Technology in Bratislava (FEI STU) in 1985 and received her $\mathrm{PhD}$ in Technical Cybernetics in 1998. Since 2013 she has been with the Institute of Automotive Mechatronics, FEI STU. Her research focus is on robust control methods in the frequency domain.

Vojtech Veselý, (Prof, DrSc) was born in Vel'ké Kapušany, Slovakia in 1940. He received MSc degree in Electrical Engineering from the Leningrad Electrical Engineering Institute, St. Peterburg, Russia, in 1964, PhD and DSc degrees from the Slovak University of Technology, Bratislava, Slovakia, in 1971 and 1985, respectively. Since 1964 he has been with the Department of Automatic Control Systems, FEI STU in Bratislava. Since 1986 he has been a full professor. His research interests include the areas of power system control, decentralized control of large-scale systems, robust control, predictive control and optimization. He is author or coauthor of more than 300 scientific papers. 